\title{
Interface excitons in type-two quantum structures
}

\author{
R. ZIMMERMANN and D. BIMBERG*
}

Max-Planck-Arbeitsgruppe "Halbleitertheorie", Hausvogteipl. 5-7, 10117 Berlin, Germany

* Institut für Festkörperphysik, Technische Universität Berlin, Hardenbergstr. 36, 10623 Berlin, Germany

\begin{abstract}
A calculation of exciton binding energies and oscillator strengths in typeII quantum structures is presented which takes into account Coulomb correlation in the growth direction, finite barrier heights, and image charge effects. Numerical results are given for excitons in InAlAs/InP superlattices and in AlAs/GaAs quantum wells. Compared with the direct exciton in type-I quantum wells, binding energies and oscillator strengths are reduced in type-II structures, but less than naively expected.
\end{abstract}

\section{BASIC THEORY}

Spatially indirect excitons in type-II (staggered line-up) structures are of increasing interest both from a fundamental point of view and for applications in photonic devices. The GaSb/InAs structure was among the first whose electronic properties were discussed in great detail [1]. Excited electrons and holes relax into adjacent layers, and the wavefunction overlap and consequently the Coulomb attraction are reduced. Following earlier work by Bastard [2], exciton effects were thus thought to be of minor importance in type-II quantum wells. We present calculations of binding energies and oscillator strengths for two type-II systems of interest, stressing the importance of Coulomb correlations in the growth direction $(z)$ which can contribute significantly to the confinement.

Even if we factorize the excitonic in-plane motion acording to

$$
\Phi\left(\vec{r}_{e}, \vec{r}_{h}\right)=U\left(z_{e}, z_{h}\right) \phi\left(\left|\vec{\rho}_{e}-\vec{\rho}_{h}\right|\right)
$$

Coulomb effects persist in the growth direction

$$
\left(-\frac{\hbar^{2}}{2 m_{e}} \partial_{z_{e}}^{2}-\frac{\hbar^{2}}{2 m_{h}} \partial_{z_{h}}^{2}+V_{e}\left(z_{e}\right)+V_{h}\left(z_{h}\right)+W\left(z_{e}-z_{h}\right)-\mathcal{E}_{x}\right) U\left(z_{e}, z_{h}\right)=0
$$

as the potential

$$
W(z)=\int_{0}^{\infty} d \rho \rho\left(\frac{\hbar^{2}}{2 \mu}\left(\phi^{\prime}(\rho)\right)^{2}-\frac{e^{2}}{\epsilon \sqrt{\rho^{2}+z^{2}}} \phi^{2}(\rho)\right)
$$

has to be added to the band edge potentials $V_{e}$ and $V_{h}$. In the present paper an exponential trial function for $\phi(\rho)$ is used. 
The two-variable Schrödinger equation (2) can be simplified introducing the factorization

$$
U\left(z_{e}, z_{h}\right) \Longrightarrow u_{e}\left(z_{e}\right) u_{h}\left(z_{h}\right)
$$

The resulting one-dimensional Schrödinger equations

$$
\begin{aligned}
\left(-\frac{\hbar^{2}}{2 m_{e}} \partial_{z_{e}}^{2}+V_{e}\left(z_{e}\right)+W_{h}\left(z_{e}\right)-E_{e}\right) u_{e}\left(z_{e}\right) & =0 \\
\left(-\frac{\hbar^{2}}{2 m_{h}} \partial_{z_{h}}^{2}+V_{h}\left(z_{h}\right)+W_{e}\left(z_{h}\right)-E_{h}\right) u_{h}\left(z_{h}\right) & =0
\end{aligned}
$$

have to be solved together since the Coulomb term

$$
W_{\nu}(z)=\int d z^{\prime} u_{\nu}^{2}\left(z^{\prime}\right) W\left(z-z^{\prime}\right) \quad(\nu=e, h)
$$

couples $u_{e}$ and $u_{h}$. To avoid double counting of the interaction, the exciton energy has to be calculated from $\mathcal{E}_{x}=E_{e}+E_{h}-W_{e h}$ where $W_{e h}$ is the average of $W\left(z_{e}-z_{h}\right)$ with both eigenfunctions squared.

The different methods have been tested by calculating the binding energy of an exciton localized at a single heterointerface with infinite barriers. With the diffusion equation method of Ref. [4], (2) has been solved numerically. Due to the type-II situation, the binding energy is only a small part of the three-dimensional binding energy $\left(8 \%\right.$ at $m_{e} / m_{h}=1,11 \%$ at $\left.m_{e} / m_{h}=0.1\right)$. The factorization was found to be very close to the exact calculation, whereas the variational Ansatz according to Bastard [2]

$$
u_{e}(z)=z \exp \left(-z / b_{e}\right) \Theta(z) ; \quad u_{h}(z)=z \exp \left(z / b_{h}\right) \Theta(-z)
$$

gave unsatisfactory results (up to $10 \%$ error). We conclude that variational methods are doubtful if Coulomb correlations in the $z$-direction are important.

\section{TYPE-II EXCITONS IN InP/InAlAs}

Lattice-matched superlattices of $\mathrm{InP}$ and $\mathrm{In}_{.53} \mathrm{Al}_{.47} \mathrm{As}$ have found much attention recently. The electrons are confined to the InP layer with a band edge discontinuity of $400 \mathrm{meV}$, whereas the holes are localized in the InAlAs layer ( $300 \mathrm{meV}$ barrier). This type-II staggered bandgap behaviour has been first established by Caine et.al. [5]. Optical transitions are indirect in real space, and a drastic reduction of the oscillator strength compared with type-I structures was expected. Surprisingly, a large radiative efficiency has been reported allowing even laser emission at low temperature [6]. It is one of the aims of the present work to give theoretical arguments for the excitonic nature of this enhancement.

Using (5) we have calculated wave functions, exciton energies, and oscillator strengths for the InP/InAlAs superlattice. The band masses for both materials are taken from [7]. Fig.1 shows an example where the hole function is slightly reshaped (full curve) due to the Coulomb interaction. As a result, the electron-hole overlap increases sizably.

The squared overlap matrix element is shown in Fig.2. With decreasing InAlAs layer thickness the overlap increases dramatically since the hole is pressed more and more towards the interface where the electron wavefunction is nonzero (dotted curve). The Coulomb correlation is important for larger layer thickness only where the bandedge confinement of the hole is small (dashed curve). The scale for both curves has to be compared with the value of one attained in a type-I quantum well.

In order to get the total excitonic oscillator strength $M_{X}$ we have to multiply with the exciton wave function at zero electron-hole separation,

$$
M_{X}=\left|\phi(0) \int d z u_{e}(z) u_{h}(z)\right|^{2}
$$



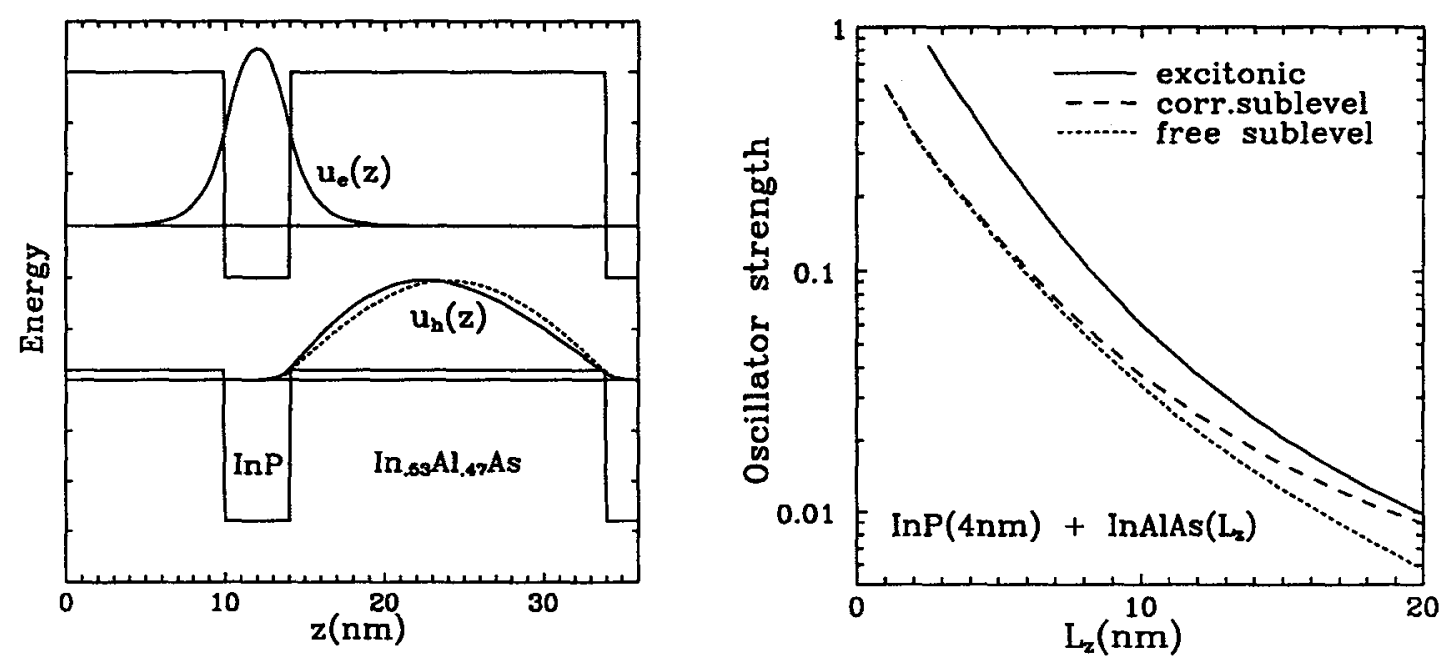

Fig.1 (left): Electron and hole wave function in a type-II superlattice (4 nm InP, $20 \mathrm{~nm}$ InAlAs). Calculations are performed with (full curve) and without (dashed curve) Coulomb correlation in the $z$ direction. The band edge discontinuities are shown schematically.

Fig.2 (right): Oscillator strength for the exciton transition (full curve) in InP/InAlAs superlattices vs. InAlAs layer width $L_{z}$. The InP layer thickness is kept fixed at $4 \mathrm{~nm}$. The squared overlap matrix element is shown with (dashed curve) and without (dotted curve) Coulomb correlation.

As seen from the full curve in Fig.2, this gives a further increase towards smaller $L_{z}$ which can be traced back to the dimensional confinement of the in-plane motion (decreasing exciton radius). The exciton binding energy increases from $3.5 \mathrm{meV}$ at $L_{z}=20 \mathrm{~nm}$ up to $7.0 \mathrm{meV}$ at $L_{z}=1 \mathrm{~nm}$.

\section{TYPE-II EXCITONS IN AlAs/GaAs}

The $X$ minimum in the AlAs barriers of AlAs/GaAs QWs is below that of GaAs. For well widths below $3.5 \mathrm{~nm}[8]$ not only the $X$ but also the $\Gamma$ minimum of GaAs is at higher energy than the AlAs $X$ minimum. Electrons near the $X$ minimum in AlAs exhibit a camel's-back dispersion leading to a large density of states and an increase of the exciton binding energy. The oscillator strength on the other hand will be low in single quantum wells due to the indirect character of the transition in real as well as in reciprocal space.

The hole is properly localized in the GaAs well, and we can use for $u_{h}\left(z_{h}\right)$ the confinement function of the first heavy-hole sublevel. In contrast, the $X$ electron is found in the AlAs material and bound by Coulombic forces only. Therefore, its wave function has to be determined from Eq.(5). A related problem deals with type-1 QWs having a valence band offset near to zero, as for $\mathrm{CdMnTe} / \mathrm{CdTe}$. The variational approach [9] was improved by a full numerical solution of the hole confinement by $\mathrm{Wu}[10]$, which in the present context is of the "factorization level".

In contrast to the InP/InAlAs system, the difference in the dielectric constants between GaAs $\left(\epsilon_{W}=12.53\right)$ and AlAs $\left(\epsilon_{B}=10.06\right)$ cannot be neglected. We have included image potential effects as shown in detail in Ref.[11].

Due to their strong mass anisotropy, the different $X$ minima are not equivalent. In our calculations we found the $X_{z}$ exciton always more tightly bound: For $L_{z}=2 \mathrm{~nm}$ we obtained an $X_{z}$ binding energy of $9.9 \mathrm{meV}$ and an exciton radius of $9.7 \mathrm{~nm}$. The corresponding $X_{x y}$ exciton values are $E_{B}=$ $4.1 \mathrm{meV}$ and $a_{x}=15.0 \mathrm{~nm}$, respectively. These values have to be compared with the direct exciton at 
the $\Gamma$ point: $E_{B}=16.6 \mathrm{meV}, a_{x}=8.6 \mathrm{~nm}$. As expected, the direct exciton is bound much stronger since electron and hole are both confined in the well.

For the indirect transition in k-space, Eq.(8) for the oscillator strength has to be complemented by a phase factor which takes into account the symmetry of the underlying Bloch functions, at least approximately. For the $X_{z}$ transition, $\exp \left(i Q_{z} z\right)$ has to be inserted, where $Q_{z}$ is the momentum difference between the $\Gamma$ point and the $X$ point. In contrast to the bulk, a finite result is obtained [12] since the translational symmetry in the $z$ direction is broken. However, the numerical value of the matrix element is extremely small (for $L_{z}=2 \mathrm{~nm}$, we found $1.2 \cdot 10^{-5}$ of the direct $\Gamma$ exciton value). Therefore rather long radiative lifetimes are expected in agreement with experimental findings $[8,13]$, and phonon-assisted recombination has to be taken into account. No-phonon excitonic transitions can be seen if interface roughness provides the necessary wave vector transfer [14].

\section{References}

[1] Sai-Halasz G.A., Esaki L. and Harrison W.A., Phys. Rev. B 18 (1978) 2812; Voisin P., Bastard G. and Gonzalves da Silva C.E.T., Solid State Commun. 39 (1981) 79.

[2] Bastard G., Phys. Rev. B 24 (1981) 4714; and Wave mechanics applied to semiconductor heterostructures (Les Editions de Physique, Les Ulis, 1988) p. 139.

[3] Duggan G. and Ralph H.I., SPIE 792 (1987) 147.

[4] Press W.H., Flannery B.P., Teukolsky S.A. and Vetterling W.T., Numerical Recipes (University Press, Cambridge, 1986) Chap. 17.2.

[5] Caine E.J., Subanna H., Kroemer H., Merz J.L. and Cho A.Y., Appl. Phys. Lett. 45 (1984) 1123.

[6] Lugagne-Delpon E., Andre J.P., Voisin P. and Voos M., Proc. 21st ICPS Beijing, Eds. Piang Jiang and Hou-Zhi Zheng (World Scientific, Singapore, 1993), p. 1000.

[7] Madelung O., Schulz M. and Weiss H. (Eds.), Landolt-Börnstein, New Series III/17a (Springer, Berlin, 1982).

[8] see the review by Wilson B.A., IEEE J. Quant. Electron. 24 (1988) 1763.

[9] Chang S.K., Nurmikko A.V., Wu J.W., Kolodziejski L.A. and Gunshor R.L., Phys. Rev. B 37 (1988) 1191.

[10] Ji-Wei Wu, Solid State Commun. 67 (1988) 911.

[11] Zimmermann R. and Bimberg D., Phys. Rev. B 47 (1993) 15789.

[12] Pulsford N.J., Nicholas R.J., Dawson P., Moore K.J., Duggan G, and Foxon C.T., Phys. Rev. Lett. 63 (1989) 2284.

[13] Finkman E., Sturge M.D. and Tamargo M.C., Appl. Phys. Lett. 49 (1986) 1299.

[14] Bimberg D., Heinrichsdorff F., Bauer R.K., Gerthsen D., Stenkamp D., Mars D.E. and Miller J.N., J. Vac. Sci. Technol. B10 (1992) 1793. 